\title{
Additivity failures in mesopic brightness matching
}

\author{
Hirohisa YAGUCHI*
}

Additivity tests were carried out for a centrally-viewed $10^{\circ}$ field using heterochromatic brightness matching at 6 retinal illuminance levels from 0.01 to 1000 photopic troland (Td). The results obtained by the mix ture of $487 \mathrm{~nm}$ and $660 \mathrm{~nm}$ monochromatic stimuli showed two types of additivity failure. At the high levels of the retinal illuminance of 10,100 and $1000 \mathrm{Td}$, the brightness of the mixture was perceived to be lower than strict validity of the additivity law would predict. This is referred to as additivity failure of the reduction type. At the low levels of the retinal illuminances of 0.01 and $0.1 \mathrm{Td}$, the mixture appeared to be brighter than the brightness that the additivity law would predict. This phenomenon means additivity failure of the enhancement type. At the intermediate level of $1 \mathrm{Td}$, both types of additivity failure were seen. The type was determined by the luminance ratio of the two stimulus in the mixture. The results from another pair of mixtures, $463 \mathrm{~nm}$ and $567 \mathrm{~nm}$, showed that additivity held at low levels, additivity failure of reduction type at intermediate level, and both types of additivity failure at high levels. The additivity failure observed at the mesopic levels are attributed to interaction between rod responses and responses from the red, green, and blue cones. These interactions are complex functions of luminance level.

\section{Introduction}

The need for the establishment of a photometric system which is available for mesopic levels has been pointed out. A great deal of luminous efficiency functions for mesopic brightness have been reported. ${ }^{1) \sim 6 \text { ) Among }}$ these reports, Sagawa and Takeichi ${ }^{5)}$ have recently provided data for standardizing mesopic luminous efficiency functions. And Ikeda and Shimozono ${ }^{4)}$ have proposed a formula which represents the mesopic luminous efficiency function for brightness using both a photopic and a scotopic luminous efficiency function. The luminous efficiency function, however, gives merely information about monochromatic lights. In most cases we need to deal with polychromatic lights. In those cases the additivity of brightness of different colors becomes an annoying problem. Although Palmer) and Kokoschka $^{8)}$ proposed a model for mesopic brightness, they did not account for the additivity failure. Experimental examinations on brightness additivity have been done only at photopic levels, and those results show additivity failure.9) This failure may be accounted

* Department of Image Science and Engineering Faculty of Engineering, Chiba University, 1-33 Yayoicho, Chiba 260, Japan. for by a color vision model on the assumption that the opponent-color channels, as well as the luminance channel, contribute to the brightness perception. It may be possible that the brightness additivity would fail also at mesopic levels because of rod intrusion. As far as we know, however, there are no experimental data on additivity at mesopic levels. Quantitative data of brightness additivity are indispensable for establishing a photometric system.

In this paper, we examine the brightness additivity at mesopic levels and have obtained two types of additivity failure, i.e. reduction and enhancement.

\section{Method}

\subsection{Principle of additivity test}

The brightness additivity was measured using the method used by Yaguchi and Ikeda ${ }^{9)}$. Suppose that monochromatic lights $\lambda_{1}$ and $\lambda_{2}$ whose respective radiances are $L_{e, 01}$ and $L_{e, 02}$ are matched separately to a reference light in brightness. The mixture $\lambda_{1}$ and $\lambda_{2}$ whose radiances are $L_{e, m_{1}}$ and $L_{e, m_{2}}$ is then matched to the same reference light. We define $\rho_{1}$ and $\rho_{2}$ in the form

$$
\begin{aligned}
& \rho_{1}=L_{e, m_{1}} / L_{e, 01} \\
& \rho_{2}=L_{e, m_{2}} / L_{e, 02}
\end{aligned}
$$

$\rho_{1}$ and $\rho_{2}$ correspond to the relative amounts of brightness of $\lambda_{1}$ and $\lambda_{2}$, in the mixture 
which yields the unit brightness. The additivity can be evaluated by the term $\rho_{1}+\rho_{2}$, such that if $\rho_{1}+\rho_{2}=1$ the additivity holds, if $\rho_{1}+$ $\rho_{2}>1$ it fails as a reduction type, and if $\rho_{1}+$ $\rho_{2}<1$ it fails as an enhancement type.

\subsection{Apparatus}

The NRC Trichromator ${ }^{10)}$ which consisted of a four-channel Maxwellian view optical system was used in this experiment. Measurements were made using two of the four available channels. Each channel provided two monochromatic lights whose wavelengths were variable. The radiances of these monochromatic lights were controlled separately by neutral density wedge filters. After combining two monochromatic lights in each channel, the radiance of the mixture was controlled by a common neutral density filter. The visual field was a horizontally divided $10^{\circ}$ bipartite filed on a dark background. Two wavelengthcombinations were examined. One was a combination of $487 \mathrm{~nm}$ and $660 \mathrm{~nm}$, another was that of $463 \mathrm{~nm}$ and $567 \mathrm{~nm}$. In each wavelength-combination, a reference light was made up of two monochromatic lights of the same wavelengths as those of the test monochromatic lights. The CIE 1964 chromaticity coordinates of the reference light were $x_{10}=0.314, y_{10}=$ 0.331 . One photopic troland of the reference light was equivalent to 6.18 scotopic trolands for $487 / 660 \mathrm{~nm}$ pair and to 1.62 scotopic trolands for the $463 / 567 \mathrm{~nm}$ pair.

\subsection{Procedure}

The observer was adapted to darkness for 10 to 30 minutes before each session started. The duration of dark adaptation was set according to the retinal illuminance level of the reference light. The dimmer the reference light, the longer the dark adaptation time. Reference levels were in six log steps from 0.1 to 1000 photopic trolands (Td) for $487 / 660$ $\mathrm{nm}$ pair and 6 steps from 0.0133 to $1,330 \mathrm{Td}$ for $463 / 567 \mathrm{~nm}$. In a preliminary experiment, $L_{e, 01}$ and $L_{e, 02}$ values were approximately estimated to match a reference light. This procedure was necessary to set the neutral density wedge filters of $\lambda_{1}$ and $\lambda_{2}$ at a certain ratio of $\rho_{1}$ to $\rho_{2}$. Then the brightness of the mixture $\lambda_{1}$ and $\lambda_{2}$ was matched to the same reference light by adjusting the common neutral density wedge filter to obtain $L_{e, m_{1}}$ and $L_{e, m_{2}}$, during which the ratio of $\rho_{1}$ to $\rho_{2}$ was held to be constant. Twelve preassigned ratios of $\rho_{1}$ to $\rho_{2}$ were investigated. Among the twelve ratios, presentations of $\lambda_{1}$ alone and $\lambda_{2}$ alone and a ratio of the two which provided the same chromaticity as the reference light were included. One wavelength-combination at one retinal illuminance level was investigated in one experimental session. Five consecutive matches were carried out for each ratio using the method of adjustment. Three or five sessions under each experimental condition were carried on different days.

\subsection{Observers}

Two males, HY (the author) and TF served as observers. They have normal color visions confirmed by the Ishihara pseudo-isochromatic plates. Both observers are experienced in psychophysical experiments. Observer HY undertook all the experiments, while observer TF served only as a supplementary observer. There was little difference in the results of two observers, so the results of TF were omitted.

\section{Results and discussion}

The $\rho_{1}-\rho_{2}$ plots for six retinal illuminance levels of $487 / 660 \mathrm{~nm}$ pair are shown in Fig. 1. Each point represents the mean of three sessions (15 matches). Each error bar shows \pm 1 standard deviation of these matches. Dashed lines were drawn from the points of \pm 1 standard deviations of single presentation of $487 \mathrm{~nm}$ to those of single presentation of $660 \mathrm{~nm}$. Each filled circle indicates the result of the $\rho_{1} / \rho_{2}$ ratio which provided the same chromaticity coordinates as the reference light. At the lowest level of $0.01 \mathrm{Td}$, the data points fell in the area of $\rho_{1}+\rho_{2}<1$ indicating additivity failure of the enhancement type. The observer could see red color in test stimuli at most of $\rho_{1} / \rho_{2}$ ratios, which suggests that the cone system, probably red cone system still contributes to brightness. The red-cone contribution at the scotopic level has been also confirmed by the fact that the luminous efficiency curve at $0.01 \mathrm{Td}$ deviated from the rod's efficiency curve or the CIE scotopic efficiency function $V^{\prime}(\lambda)$ in the long wavelength region. ${ }^{4)}$ At $0.1 \mathrm{Td}$, additivity 

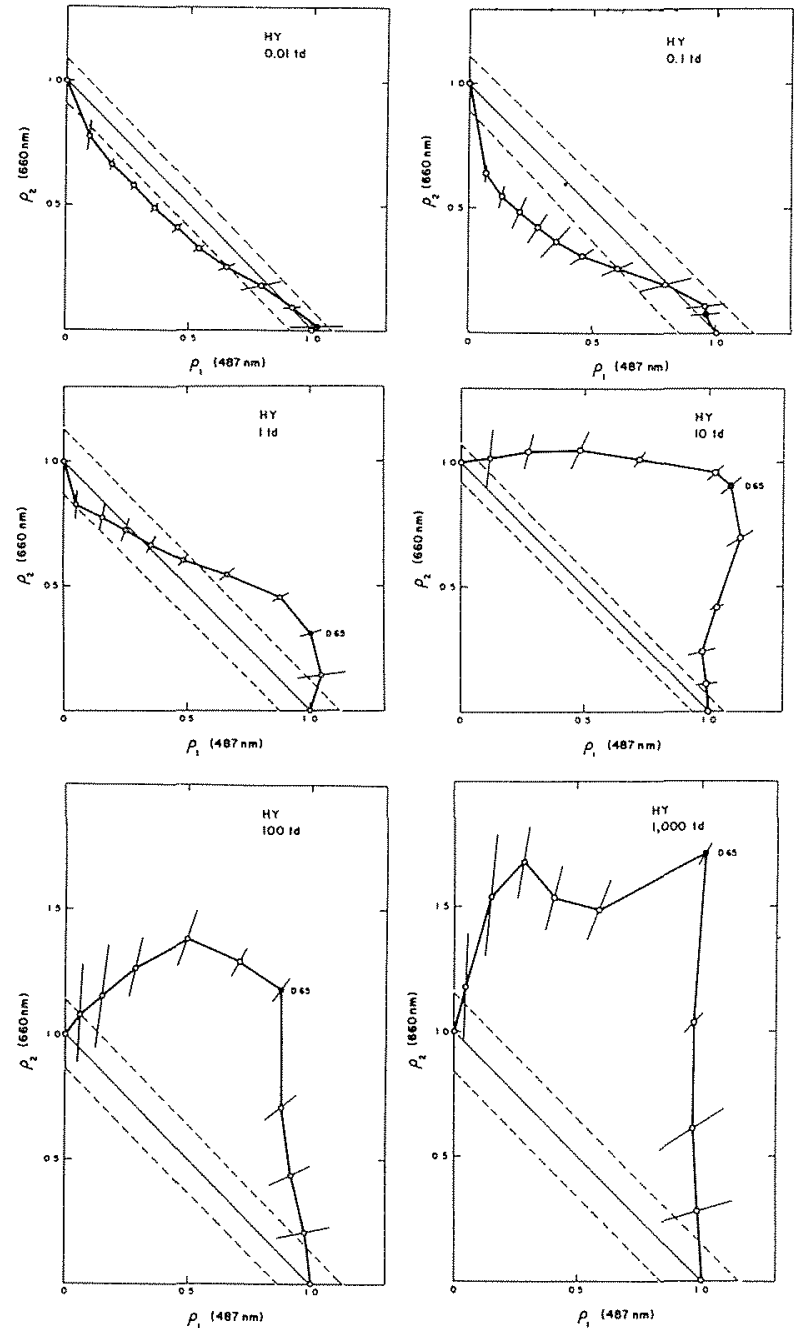

Fig. $1 \quad \rho_{1}-\rho_{2}$ plots for $487 / 660 \mathrm{~nm}$ pair of (a); $0.01 \mathrm{td}, \quad$ (b); $0.1 \mathrm{td}, \quad$ (c); .. $1 \mathrm{td}$, (d); $10 \mathrm{td}, \quad(\mathrm{e}) ; 100 \mathrm{td}$, and (f); $1000 \mathrm{td}$ from subject $H Y$. Error bars show \pm 1 $S D$ from several sessions. Closed circles indicate results obtained from test stimuli which have the same chromaticity coorinates as the reference light.

failure of enhancement type was still observed, particularly at small ratios of $\rho_{1} / \rho_{2}$. At $1 \mathrm{Td}$, the reduction type appeared at large ratios of $\rho_{1} / \rho_{2}$. At levels higher than $10 \mathrm{Td}, \rho_{1}-\rho_{2}$ plots show additivity failure of reduction type throughout the whole range of $\rho_{1} / \rho_{2}$. The degree of reduction is represented as bulge in the curve. The brightness reduction increased according as the retinal illuminance increased.

Figure 2 shows the $\rho_{1} / \rho_{2}$ plots of $463 / 567$ nm pair. The fact that at 0.0133 and $0.133 \mathrm{Td}$ levels, the data points fell on the straight line of $\rho_{1}+\rho_{2}=1$ indicates that the additivity holds at these levels. The observer reported that test stimuli appeared to be the same color
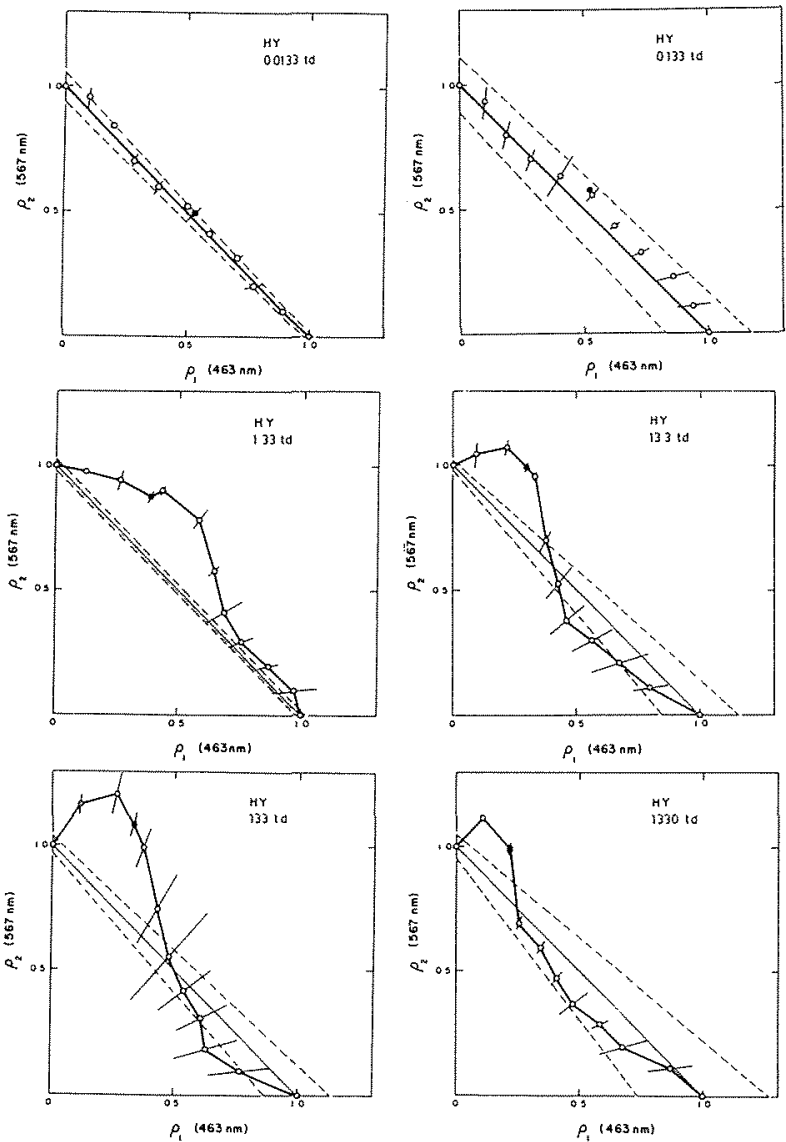

Fig. 2 Same as Fig. 1 but for $463 / 567 \mathrm{~nm}$ pair of (a); $0.0133 \mathrm{td}, \quad(b) ; 0.133 \mathrm{td}, \quad$ (c); $1.33 \mathrm{td}, \quad(d) ; 13.3 \mathrm{td},(\mathrm{e}) ; 133 \mathrm{td}$, and (f); $1330 \mathrm{td}$.

as the reference light at any $\rho_{1} / \rho_{2}$ ratios. From this fact, it can be referred that the rod system should contribute exclusively to the brightness at these levels. At $1.33 \mathrm{Td}$, the data points fell in the area of $\rho_{1}+\rho_{2}>1$ indicating additivity failure of the reduction type. It implies that the cone system in addition to the rod system contributes to brightness. At retinal illuminance levels of 13.3, 133 and 1,330 Td, a similar shape of $\rho_{1}-\rho_{2}$ curves was obtained. When the $\rho_{1} / \rho_{2}$ ratio is small, additivity failure is of the reduction type. Although the standard deviation of single presentations of $463 \mathrm{~nm}$ is large, it can be probably concluded that additivity fails as an enhancement type when the $\rho_{1} / \rho_{2}$ ratio is large.

The amount of reduction was particularly large when the $\rho_{1} / \rho_{2}$ ratio was of the same chromaticity as the reference light. In this case, the color appeared to be desaturated. Hue cancellation takes place in such a case. The coincidence of the large reduction type 
failures with desaturation of the test field implies that outputs of the opponent-color channels were at a minimum. Additivity failure of enhancement type occurred at high levels in the case of $463 / 567 \mathrm{~nm}$ pair, but occurred at low levels in the case of $487 / 660 \mathrm{~nm}$ pair. The former fact might be accounted for by the non-linear contribution of the blue-yellow opponent color channel to the brightness perception as suggested by Yaguchi et al. ${ }^{9)}$ In the latter case, however, the level is too low to activate the blue-yellow opponent color channel. It might be caused by the interaction between rods and long wavelength sensitive cones (red cones). Frumkes et al $^{11)}$ suggested that the interaction between rods and cones was inhibitory at the detection of increment difference, but the present results show brightness enhancement. This kind of inconsistency also occurs in rod free conditions. Increment threshold experiments show additivity failure of the reduction type only, but the heterochromatic brightness matching shows additivity failure of both types. ${ }^{12)}$ It could be that the mechanism of detection of increment difference and the criterion of brightness matching are somehow different from one another.

Although a few models of mesopic brightness prediction have been proposed, none of them can account for the present results. Palmer ${ }^{7}$ proposed a photometric model which covered the scotopic level. In his model, luminance at the mesopic level is presented as a nonlinear equation using the scotopic luminance and the photopic luminance. His model, however, cannot explain additivity failure of the enhancement type. Kokoschka et $\mathrm{al}^{8)}$ proposed a model in which luminance is presented as a linear function of the CIE tristimulus values and scotopic luminance. Since the model assumes a linear function, it always holds for the additivity law. Yaguchi and Ikeda ${ }^{9)}$ accounted for additivity failure of both types by a non-linear vector model on the assumption that the opponent-color channel outputs were non-linearly transferred to brightness perception, but this could be restricted only to photopic levels. If rod outputs as well as opponent-color outputs are applied to this model, the present results should be fitted. But the present results contain insufficient data to confirm the validity of this model. In order to establish a new mesopic photometric model, we still need more data on the additivity examination of mesopic brightness.

\section{Acknowledgments}

This work was done at National Research Council of Canada while the author was staying there as a Research Associate. He would like to thank the late Dr. G. Wyszecki and Dr. W.B. Cowan for many helpful discussions and $\mathrm{Mr}$. T. Fuchida for his help as an observer.

\section{References}

(1) Sloan, L.L.: "The effect of intensity of light, state of adaptation of the eye, and the size of photometric field on the visibility curve", Psychol. Monogr. 38 (1928) 1 87.

(2) Walters, H.V. and Wright, W.D.: "The spectral sensitivity of the fovea and extra-fovea in the Purkinje range", Proc. R. Soc. Lond., Ser. B, 131 (1984) 340 361.

(3) Kinney, J.A.S.: "Comparison of scotopic, mesopic, and photopic spectral sensitivity curves", J. Opt. Soc. Am., 48 (1958) 185 190.

(4) Ikeda, M. and Shimozono, H.: 'Mesopic luminous-efficiency functions", J. Opt. Soc. Am 71 (1981) 280 284.

(5) Sagawa, K. and Takeichi, K.: "Luminous efficiency function for mesopic vision", CIE 20 th Session '83, D103/1-4 (1983).

(6) Yaguchi, H. and Ikeda, M.: "Mesopic luminousefficiency functions for various adapting levels", J. Opt. Soc. Am. A-1 (1984) 120 123.

(7) Palmer, D.A.: "Standard observer for largefield photometry at any level", J. Opt. Soc. Am. 58 (1968) 1296 1299.

(8) Kokoschka, S. and Bodmann, H.W.: "Ein konsistentes System zur photometrischen Strahlungs bewertung im gesamten Adaptationsbereich", CIE 18th Session '75, P-75-14, (1975) 217 225.

(9) Yaguchi, H. and Ikeda, M.: "Subadditivity and superadditivity in heterochromatic brightness matching", Vision Res. 23 (1983) 1711 1718.

(10) Wyszecki, G. and Stiles, W.: Color Science, 2nd edition, Wiley, New York, (1982) 476 478.

(11) Frumkes, T.E., Sekuler, M.D. and Reiss, E.H.: "Rod-cone interaction in human scotopic vision", Science, 175 (1972) 913 914.

(12) Guth, S.L., Donley, N.J., and Marrocco, R.T.: "On luminance additivity and related topics", Vision Res. 9 (1969) 537 575.

Received 1 Aug. 1986: Revision Received 30 Oct. 1986. 